\title{
Continuous High-Frequency Oscillation Therapy in Invasively Ventilated Pediatric Subjects in the Critical Care Setting
}

\author{
Stephen Morgan RRT, Christoph P Hornik MD MPH, Niyati Patel MD, Walter L Williford RRT, \\ David A Turner MD, and Ira M Cheifetz MD FAARC
}

\begin{abstract}
BACKGROUND: Continuous high-frequency oscillation (CHFO) creates a pressure gradient in the small airways that accelerates expiratory flow. The intended use of CHFO therapy is to facilitate secretion removal and treat atelectasis. Our objective was to assess the feasibility, safety, and efficacy of CHFO in the mechanically ventilated pediatric population. METHODS: After institutional review board approval, we retrospectively reviewed medical records of mechanically ventilated children treated with CHFO (the MetaNeb system) at our institution from July 1, 2007 through August 31, 2012. Patients supported with extracorporeal membrane oxygenation were excluded. We evaluated changes in ventilator settings in subjects with ventilator data documented within $6 \mathrm{~h}$ pre- and post-treatment. We evaluated arterial blood gas (ABG) results for individual treatments, comparing ABG results within $8 \mathrm{~h}$ pre-therapy to ABG results within $3 \mathrm{~h}$ post-treatment. Oxygen index and $\mathrm{P}_{\mathrm{aO}} / \mathrm{F}_{\mathrm{IO}}$, were calculated. Demographic data, blood pressure, heart rate, and development of new air leak while being treated with CHFO were recorded. Pre- and post-CHFO measurements were compared using Wilcoxon signed-rank testing. RESULTS: Our cohort included 59 invasively ventilated subjects. Median age was 2 y (range 1 month to $19 \mathrm{y})$, and median weight was $14 \mathrm{~kg}(2-81 \mathrm{~kg})$. We evaluated data on 528 total treatments (range per subject 1-39 treatments). Peak inspiratory pressure significantly decreased with CHFO, whereas other parameters, including $\mathrm{P}_{\mathrm{aCO}}$ and breathing frequency, remained stable. There was no significant change in systolic blood pressure, diastolic blood pressure, or heart rate following treatment with CHFO. One subject (2\%) developed a clinically insignificant pneumothorax during CHFO. CONCLUSIONS: CHFO is feasible and seems safe in our cohort of mechanically ventilated pediatric subjects. The rate of pneumothorax was consistent with that seen in similar pediatric ICU populations. These preliminary results suggest that CHFO may be beneficial by improving lung compliance in pediatric subjects with secretion-induced atelectasis. Prospective clinical studies are needed to further evaluate the clinical efficacy and safety of CHFO in children receiving invasive mechanical ventilation. Key words: atelectasis; intrapulmonary percussive ventilation; mucociliary clearance; airway clearance; pediatric; gas exchange; mechanical ventilation. [Respir Care 2016;61(11):1451-1455. (C) 2016 Daedalus Enterprises]
\end{abstract}

\section{Introduction}

As many as $10 \%$ of the estimated 115,000 mechanically ventilated infants and children in the United States each

\footnotetext{
Mr Morgan and Dr Cheifetz are affiliated with Respiratory Care Services, Duke University Hospital, Duke University Medical Center, Durham, North Carolina. Drs Hornik, Turner, and Cheifetz are affiliated with the Division of Pediatric Critical Care Medicine, and Dr Hornik is affiliated with the Division of Quantitative Sciences, Department of Pediatrics, Duke Children's Hospital, Duke University Medical Center, Durham, North Carolina. Dr Patel is affiliated with the Division of Pediatric Critical Care Medicine, Department of Pediatrics, University of Minnesota, Minneapolis, Minnesota. Mr Williford is affiliated with Respiratory Care Services, Children's Hospital of the King's Daughters, Norfolk, Virginia.
}

year develop atelectasis. ${ }^{1,2}$ Risk factors for atelectasis in ventilated neonatal and pediatric patients include small, collapsible airways, a compliant chest wall, and the absence of intra-alveolar and bronchoalveolar pores. ${ }^{3}$ Additional risk factors for atelectasis, which may be present in

\footnotetext{
This work was supported in part by a research grant from Hill-Rom. Dr Cheifetz has disclosed relationships with Philips and Ikaria.

Correspondence: Ira M Cheifetz MD FAARC, Division of Pediatric Critical Care Medicine, Duke University Medical Center, Box 3046, Durham, NC 27710. E-mail: ira.cheifetz@duke.edu.
}

DOI: $10.4187 /$ respcare.04368 


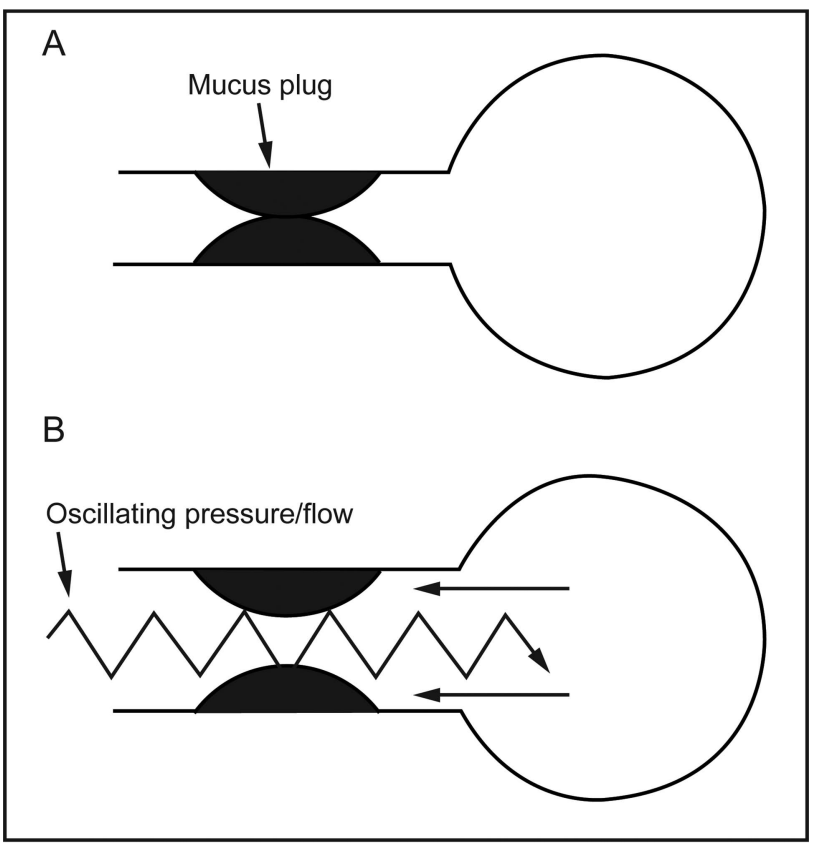

Fig. 1. Mechanism of intrapulmonary percussive ventilation. Adapted from PercussiveNeb user's guide.

critically ill patients, include disruption of the mucociliary escalator and ineffective cough. These changes may lead to difficulty with secretion clearance that may cause airway obstruction and accumulation of secretions, ${ }^{4}$ potentially increasing the risk of ventilator-associated pneumonia. ${ }^{5}$ Inadequate secretion clearance can also create secondary areas of lung hyperexpansion. Untreated atelectasis and impaired mucociliary clearance may result in prolonged duration of mechanical ventilation and, ultimately, a prolonged pediatric ICU stay.

In an effort to potentially improve therapy for atelectasis, intrapulmonary percussive ventilation (IPV) was introduced in 1985 by Dr Forrest Bird as an alternative treatment for atelectasis and secretion clearance. Continuous high-frequency oscillation (CHFO) is similar to IPV in that both technologies deliver high-flow minibursts of air to the lungs ${ }^{6}$ and are designed for use either in conjunction with a conventional ventilator or as a stand-alone device.

CHFO creates a mean airway pressure that helps to prevent premature closure of lung regions while actively recruiting already collapsed areas. In addition, the continuous sub-tidal volume oscillation creates a cephalad counterflow of air that promotes upward movement of secretions (Fig. 1). The intended use of CHFO therapy is to facilitate secretion removal and treat atelectasis.

Despite the importance of atelectasis prevention and secretion clearance in mechanically ventilated patients, data addressing the optimal treatment for atelectasis are lacking. To date, evidence for CHFO or IPV as effective therapies for atelectasis remains limited to case reports and

\section{QUICK LOOK}

\section{Current knowledge}

Continuous high-frequency oscillation (CHFO) creates a pressure gradient in the small airways that accelerates expiratory flow. Gas flow associated with CHFO therapy may facilitate secretion removal and treat atelectasis.

\section{What this paper contributes to our knowledge}

CHFO is feasible and seems safe in mechanically ventilated pediatric patients, within the modes and ventilatory approaches used in this group of subjects. These preliminary results suggest CHFO may be beneficial by improving lung compliance in pediatric patients with secretion-induced atelectasis.

retrospective studies. ${ }^{7-10}$ Published evidence for the use of CHFO in invasively ventilated pediatric patients is lacking. Thus, our objective was to assess the feasibility, safety, and efficacy of CHFO in the mechanically ventilated pediatric population.

\section{Methods}

\section{Subjects}

We retrospectively reviewed the medical records of all invasively ventilated infants and children treated with CHFO at our institution from January 1, 2007 through August 31, 2012. Exclusion criteria included those patients supported with extracorporeal life support (eg, extracorporeal membrane oxygenation). CHFO was employed at clinician discretion using the MetaNeb system (Hill-Rom, Batesville, Indiana). It should be noted that the device was used off label because the device is currently FDA-cleared for use in patients $\geq 2$ y of age. All subjects were ventilated with the AVEA ventilator (CareFusion, Yorba Linda, California). A spring-loaded T with a one-way valve was placed on the inspiratory limb of the ventilator circuit. The subject was positioned with the head of bed elevated at $>30^{\circ}$ unless contraindicated. MetaNeb mode was set to $\mathrm{CHFO}$ with the higher option selected. The green selector ring from the subject end of the handset was removed and replaced with the black occlusion ring, ensuring that the exhalation orifice was occluded. A nebulizer adapter with a one-way valve and a $15-\mathrm{mm}$ adapter were used to connect the MetaNeb to the spring-loaded T placed in the inspiratory limb.

The use of nebulized medications, including albuterol, $\mathrm{N}$-acetylcysteine, and dornase alfa, administered via the 
MetaNeb system was not directed by protocol. For medication delivery, an Aero Eclipse nebulizer (Trudell Medical International, London, Canada) in continuous mode was used. Standard medication dosing was applied with a maximum nebulizer volume of $6 \mathrm{~mL}$. The treatment duration of medication delivery was approximately $10 \mathrm{~min}$. The study was approved by the institutional review board at Duke University Medical Center, and the requirement for informed consent was waived.

\section{Data Collection}

Electronic medical records were reviewed for each patient admitted to our pediatric ICU or pediatric cardiac ICU who received CHFO while being invasively ventilated. Clinical data included heart rate, breathing frequency, blood pressure, arterial blood gases, and ventilator parameters (peak inspiratory pressure and tidal volume). Baseline data were recorded as the most recent arterial blood gas and most recent documented ventilator parameters within $12 \mathrm{~h}$ of initiation of CHFO therapy. Subsequent data were obtained before and after each CHFO episode, comparing ventilator data documented within $6 \mathrm{~h}$ pre- and post-treatment and comparing arterial blood gas (ABG) results within $8 \mathrm{~h}$ pretherapy with $\mathrm{ABG}$ results within $3 \mathrm{~h}$ post-treatment. Complications were recorded, including new development or worsening of pneumothorax, pneumomediastinum, or other air leak.

\section{Data Analysis}

We used medians and ranges to describe continuous variables and counts and percentages for categorical variables. Hemodynamic, blood gas, and ventilator data were compared pre- and post-CHFO therapy using the nonparametric Wilcoxon signed-rank test. We considered $P<.05$ statistically significant and conducted all analyses using Stata 13.1 (Stata Corp, College Station, Texas).

\section{Results}

\section{Subjects}

Fifty-nine pediatric subjects were studied, of which $63 \%$ were male. These subjects had a median age of 2 y (range 1 month to $19 \mathrm{y})$ and a median weight of $14 \mathrm{~kg}(2-81 \mathrm{~kg})$. Demographic data are shown in Table 1. This patient series includes 528 CHFO treatments (1-39 treatments/subject). Seventy-five percent of these subjects received $\leq 10$ CHFO treatments.

Eighty percent of the subjects were supported with pressure control/synchronized intermittent mandatory ventilation + pressure support, $10 \%$ with pressure-regulated volume control ventilation, and $10 \%$ with other modes of
Table 1. Distribution of Subject Age and Clinical Diagnoses

\begin{tabular}{lrr}
\hline \hline \multicolumn{1}{c}{ Characteristics } & Total $(N=59)$ & Percentage \\
\hline Age, y & 25 & \\
$<2$ & 14 & 42 \\
$2-5$ & 9 & 25 \\
$6-10$ & 11 & 15 \\
$>10$ & & 18 \\
Primary diagnosis & 14 & \\
Post-op cardiac & 10 & 24 \\
Medical cardiac & 17 & 17 \\
Respiratory & 3 & 29 \\
Hematology/oncology & 11 & 5 \\
Neurology/neurosurgical & 4 & 19 \\
Other non-cardiac & & 5 \\
Ethnicity & 37 & \\
White/Caucasian & 17 & 63 \\
African-American & 4 & 29 \\
Hispanic/Latino & 1 & 7 \\
Asian & & 1 \\
\hline
\end{tabular}

ventilation (pressure support or airway pressure release ventilation). Ventilator and gas exchange parameters are shown in Table 2. No significant change in systolic blood pressure, diastolic blood pressure, or heart rate was seen following treatment with CHFO (data not shown). One subject (2\%) developed an air leak following initiation of CHFO.

Peak inspiratory pressure significantly decreased (14 [IQR 12-18] $\mathrm{cm} \mathrm{H}_{2} \mathrm{O}$ versus 8 [IQR 6-11] $\mathrm{cm} \mathrm{H}_{2} \mathrm{O}$, $P<.001)$ following CHFO therapy. $\mathrm{F}_{\mathrm{IO}_{2}}$ was slightly higher (0.35 [IQR 0.30-0.40] vs 0.40 [IQR 0.35-0.45], $P=.002)$, and $\mathrm{S}_{\mathrm{aO}_{2}} / \mathrm{F}_{\mathrm{IO}_{2}}$ was slightly lower (259 [IQR 210-313] versus 243 [IQR 198-277], $P=.003$ ) following CHFO, but these changes do not appear clinically important. $\mathrm{pH}$ following CHFO was slightly lower (7.41 [IQR 7.36-7.46] versus 7.39 [IQR 7.35-7.43], $P=.001$ ) but remained in the normal range. Other parameters, including $\mathrm{P}_{\mathrm{aCO}_{2}}, \mathrm{P}_{\mathrm{aO}_{2}} / \mathrm{F}_{\mathrm{IO}_{2}}$, oxygenation index, exhaled tidal volume, and breathing frequency showed no significant changes with CHFO therapy.

\section{Discussion}

Thousands of children who are mechanically ventilated in the United States each year develop atelectasis. Atelectasis may prolong the duration of mechanical ventilation and increase the risk of ventilator-associated pneumonia, both of which are sources of morbidity and prolonged hospitalization. ${ }^{5}$ Additionally, impaired mucociliary clearance leads to areas of plugging and hyperexpansion, resulting in increased dead space and resultant increased risk of extubation failure. ${ }^{11}$ For this reason, treatment of atel- 
Table 2. Ventilator and Gas Exchange Data

\begin{tabular}{|c|c|c|c|c|}
\hline & No. of Treatments (Pre/Post) & Pre-CHFO Therapy & Post-CHFO Therapy & $P$ \\
\hline $\mathrm{PIP}, \mathrm{cm} \mathrm{H}_{2} \mathrm{O}$ & $479 / 503$ & $14(12-18)$ & $8(6-11)$ & $<.001$ \\
\hline Exhaled tidal volume, $\mathrm{mL}$ & $509 / 520$ & $110(73-190)$ & $106(70-210)$ & .82 \\
\hline PEEP, $\mathrm{cm} \mathrm{H}_{2} \mathrm{O}$ & $517 / 523$ & $7(6-10)$ & $7(6-10)$ & .31 \\
\hline Mean airway pressure, $\mathrm{cm} \mathrm{H}_{2} \mathrm{O}$ & $516 / 523$ & $11(9-14)$ & $12(10-14)$ & .11 \\
\hline $\mathrm{F}_{\mathrm{IO}_{2}}$ & $517 / 524$ & $0.35(0.30-0.40)$ & $0.40(0.35-0.45)$ & .002 \\
\hline Breathing frequency, breaths/min & $514 / 522$ & $21(16-30)$ & $22(17-30)$ & .06 \\
\hline $\mathrm{pH}$ & $448 / 528$ & $7.41(7.36-7.46)$ & $7.39(7.35-7.43)$ & $<.001$ \\
\hline $\mathrm{P}_{\mathrm{aCO}_{2}}, \mathrm{~mm} \mathrm{Hg}$ & $448 / 528$ & $44(38-50)$ & $43(39-48)$ & .50 \\
\hline Oxygen index & $444 / 523$ & $4.8(3.0-8.8)$ & $5.2(3.2-8.8)$ & .23 \\
\hline $\mathrm{S}_{\mathrm{aO}_{2}} / \mathrm{F}_{\mathrm{IO}_{2}}$ & $438 / 523$ & $259(210-313)$ & $243(198-277)$ & .003 \\
\hline $\begin{array}{l}\text { Values are medians (IQR). } \\
\text { CHFO = continuous high-frequency oscillation } \\
\text { PIP = peak inspiratory pressure }\end{array}$ & & & & \\
\hline
\end{tabular}

ectasis and effective augmentation of mucociliary clearance are important in mechanically ventilated children.

Although data are limited, previous studies suggest that CHFO and similar therapies may be effective for secretion clearance and treatment of atelectasis. ${ }^{11-13}$ Deakins and Chatburn ${ }^{12}$ conducted a retrospective analysis to evaluate the impact of IPV in 46 mechanically ventilated pediatric subjects. Following the retrospective analysis, a randomized controlled trial was conducted and reported as part of the same publication. ${ }^{12}$ In this study, 12 subjects were randomly assigned to receive either IPV therapy or standard chest physiotherapy. In both phases of their study, the authors assessed the impact of atelectasis on chest radiograph scores. In the retrospective phase of the study, a statistically significant improvement in atelectasis scores was found following treatment with IPV therapy. In the prospective portion, a statistically significant improvement in atelectasis score was seen in the IPV-treated group but not in the group receiving standard chest physiotherapy. Additionally, time of treatment to the resolution of atelectasis was significantly less in the IPV group as compared with the chest physiotherapy group. ${ }^{12}$

Our retrospective study was conducted with the primary aim of assessing the feasibility, effectiveness, and safety of CHFO in a heterogeneous population of infants and children requiring invasive mechanical ventilation. The impact on ventilator and gas exchange parameters in a population of critically ill patients has not been studied previously.

The current study, although underpowered, represents the largest reported sample of such subjects. CHFO therapy was well tolerated in a population of mechanically ventilated pediatric patients. Vital signs remained stable, and the rate of pneumothorax was consistent with that seen in similar pediatric ICU populations. ${ }^{13,14}$ In addition, peak inspiratory pressure significantly decreased following
CHFO therapy, whereas other parameters, including $\mathrm{P}_{\mathrm{aCO}_{2}}$ and breathing frequency, remained stable.

In our pediatric ICU, patients are typically ventilated in pressure ventilation modes. In the current study, the majority $(80 \%)$ of subjects were supported with pressure control/synchronized intermittent mandatory ventilation + pressure support. As such, the results suggest that weaning of peak pressure was possible following CHFO therapy, although it is possible that the decrease in peak pressure could have occurred without the therapy. The dramatic change that we observed in the short time following therapy, however, suggests that the intervention may have contributed to the result.

The retrospective design has inherent limitations, and our study was not designed or adequately powered for efficacy or safety. Larger, prospective clinical studies are needed to fully evaluate CHFO therapy in children receiving invasive mechanical ventilation. Despite the limitations, however, our data support the feasibility and apparent safety of CHFO in mechanically ventilated infants and children. Our data also suggest that CHFO may be beneficial by improving lung compliance in pediatric patients with secretion-induced atelectasis. Further investigation with a prospective study is needed to confirm (or refute) our findings.

\section{Conclusions}

CHFO is feasible and seems safe in our cohort of mechanically ventilated pediatric subjects, within the modes and ventilatory approaches used in this group of subjects. The rate of pneumothorax is consistent with that seen in similar pediatric ICU populations. These preliminary results suggest CHFO may be beneficial by improving lung compliance in pediatric patients with secretion-induced atelectasis. Prospective clinical studies are needed to further 


\section{CHFO in Invasively Ventilated Pediatric Subjects}

evaluate the clinical efficacy and safety of CHFO in children receiving invasive mechanical ventilation.

\section{REFERENCES}

1. Rivera R, Tibballs J. Complications of endotracheal intubation and mechanical ventilation in infants and children. Crit Care Med 1992; 20(2):193-199.

2. Watson SR, Hartman ME. Epidemiology of Critical Illness. In: Wheeler DS, Wong HR, Shanley TP, editors. Pediatric critical care medicine: basic science and clinical evidence. London: Springer; 2007:31-36.

3. Peroni DG, Boner AL. Atelectasis: mechanisms, diagnosis and management. Paediatr Respir Rev 2000;1(3):274-278.

4. Gal TJ. Effects of endotracheal intubation on normal cough performance. Anesthesiology 1980;52(4):324-329.

5. Marini JJ, Pierson DJ, Hudson LD. Acute lobar atelectasis: a prospective comparison of fiberoptic bronchoscopy and respiratory therapy. Am Rev Respir Dis 1979;119(6):971-978.

6. McInturff S, Shaw L, Hodgkin J, Rumble L, Bird F. Intrapulmonary percussive ventilation (IPV) in the treatment of COPD. Respir Care 1985;30(10):885.

7. Nino G, McNally P, Miske LJ, Hickey E, Panitch HB. Use of intrapulmonary percussive ventilation (IPV) in the management of pulmonary complications of an infant with osteogenesis imperfecta. Pediatr Pulmonol 2009;44(11):1151-1154.
8. Toussaint M, De Win H, Steens M, Soudon P. Effect of intrapulmonary percussive ventilation on mucus clearance in duchenne muscular dystrophy patients: a preliminary report. Respir Care 2003; 48(10):940-947.

9. Tsuruta R, Kasaoka S, Okabayashi K, Maekawa T. Efficacy and safety of intrapulmonary percussive ventilation superimposed on conventional ventilation in obese patients with compression atelectasis. J Crit Care 2006;21(4):328-332.

10. Wada N, Murayama K, Kaneko T, Kitazumi E. [Effect of intrapulmonary percussive ventilation in a severely disabled patient with persistent pulmonary consolidation]. No To Hattatsu 2005;37(4): 332-336.

11. Hubble CL, Gentile MA, Tripp DS, Craig DM, Meliones JN, Cheifetz IM. Deadspace to tidal volume ratio predicts successful extubation in infants and children. Crit Care Med 2000;28(6): 2034-2040.

12. Deakins K, Chatburn RL. A comparison of intrapulmonary percussive ventilation and conventional chest physiotherapy for the treatment of atelectasis in the pediatric patient. Respir Care 2002;47(10): 1162-1167.

13. Principi T, Fraser DD, Morrison GC, Farsi SA, Carrelas JF, Maurice EA, Kornecki A. Complications of mechanical ventilation in the pediatric populations. Pediatr Pulmonol 2011;46(5):452-457.

14. da Silva PS, de Aguiar VE, Fonseca MC. Iatrogenic pneumothorax in mechanically ventilated children: Incidence, risk factors and other outcomes. Heart and Lung 2015;44(3):238-242.

This article is approved for Continuing Respiratory Care Education credit. For information and to obtain your CRCE

(free to AARC members) visit

www.rcjournal.com 